\title{
Three-dimensional measurement and analysis of morphological parameters of the uterus in infertile women
}

\author{
Yankun Feng $^{1 \#}$, Shaojing Zhang ${ }^{2 \#}$, Ying Zhou ${ }^{2 \#}$, Guibing He ${ }^{3}$, Liting Hong ${ }^{1}$, Li Shi ${ }^{1}$, Jianmei Wang ${ }^{2}$, \\ Ping Zhang ${ }^{1}$, Lidong Zhai ${ }^{1}$ \\ ${ }^{1}$ Department of Anatomy and Histology, School of Basic Medical Sciences, Tianjin Medical University, Tianjin, China; ${ }^{2}$ Department of Family \\ Planning, The Second Hospital of Tianjin Medical University, Tianjin, China; ${ }^{3}$ Department of Urology, Jinhua People’s Hospital, Jinhua, China
}

Contributions: (I) Conception and design: Y Feng, S Zhang, Y Zhou, J Wang, L Zhai; (II) Administrative support: S Zhang, J Wang, P Zhang; (III) Provision of study materials or patients: S Zhang, Y Zhou, J Wang; (IV) Collection and assembly of data: Y Feng, Y Zhou, S Zhang, L Hong, L Shi, J Wang, L Zhai; (V) Data analysis and interpretation: Y Feng, G He, L Zhai; (VI) Manuscript writing: All authors; (VII) Final approval of manuscript: All authors.

\#These authors contributed equally to this work.

Correspondence to: Lidong Zhai. Department of Anatomy and Histology, School of Basic Medical Sciences, Tianjin Medical University, 22 Qixiangtai Road, Heping District, Tianjin 300070, China. Email: zhailidong2005@126.com; Jianmei Wang. Department of Family Planning, The Second Hospital of Tianjin Medical University, 23 Pingjiang Road, Tianjin 300211, China. Email: wangjianmei@tmu.edu.cn.

Background: To determine differences in endometrial cavity anteroposterior diameter, thickness, volume, and diameter lines of uterine body and thickness, and volume of upper, middle, and lower regions of the endometrium in infertile women using a new method for three-dimensional (3D) reconstruction based on two-dimensional (2D) ultrasound images.

Methods: This retrospective cross-sectional study included a total of 81 infertile women, who underwent 2D ultrasound standard examination. We created 3D models of the uterine body, endometrial cavity, and endometrium based on 2D ultrasound images. The parameters that were measured and analyzed in a 3D plane included volume and diameter lines of endometrial cavity, surface area, thickness, volume, and diameter lines of uterine body, and surface area, thickness, and volume of upper, middle, and lower region of the endometrium. These parameters were used for comparisons between normal and arcuate uterus, between non-pregnant and pregnant infertile women, and between nulliparous and multiparous infertile women. The differences between the different regions of the endometrium and the correlations between age and the parameters were also determined in this study.

Results: Endometrial cavity length, and middle and lower regions of the endometrial volume in the normal uterus were $39.63 \pm 7.61 \mathrm{~mm}, 1,307.92 \pm 1,034.40 \mathrm{~mm}^{3}$, and $653.98 \pm 460.41 \mathrm{~mm}^{3}$, respectively. For arcuate uterus, these parameters were $32.96 \pm 4.69 \mathrm{~mm}, 539.89 \pm 298.94 \mathrm{~mm}^{3}$, and $347.90 \pm 129.61 \mathrm{~mm}^{3}$, respectively. The parameters were significantly higher in normal uterus compared with arcuate uterus $(\mathrm{P}=0.000,0.001$, and 0.006, respectively). Upper, middle, and lower regions of endometrial thickness in normal uterus were $7.79 \pm 3.26,8.18 \pm 3.33$, and $6.41 \pm 2.60 \mathrm{~mm}$, respectively. Both upper and middle regions of endometrial thickness were significantly greater than the lower regions of endometrial thickness with $\mathrm{P}=0.009$ and $\mathrm{P}=0.001$, respectively. Correlation analysis revealed that age positively correlated with volume of upper endometrial regions ( $\mathrm{r}=0.274, \mathrm{P}=0.028)$.

Conclusions: This study provides references for the volume and thickness of the endometrium in the different anatomical regions of normal and arcuate uterus. Age mainly affects the upper region of the endometrium. The $3 \mathrm{D}$ measurement provides a precise way to quantify the morphological parameters of gynecological diseases. 
Keywords: Three-dimensional measurement (3D measurement); infertile women; uterine body; endometrial cavity; endometrium

Submitted Aug 15, 2021. Accepted for publication Dec 13, 2021; Published online: 06 Jan 2022.

doi: $10.21037 /$ qims-21-812

View this article at: https://dx.doi.org/10.21037/qims-21-812

\section{Introduction}

Although morphological parameters of the uterus and its related structures have been extensively measured and studied in normal females (1-5), uterine body thickness (UBT) has not yet been described. To date, few authors have reported measurements of endometrial cavity anteroposterior diameter (ECA). Goldstuck studied the ECA, which was derived from the ellipsoid formula (6); however, the results of their study were not exact, because the shape of the uterine cavity is not an ellipse. The endometrium is central to female reproductive physiology (7). To date, morphological features of the endometrium in different anatomical regions have not been investigated. In recent years, given research developments, structural features of the uterus in infertile women have been gradually investigated $(8,9)$. However, these studies have mostly focused on the uterus, while little attention has been paid to the uterine body, endometrial cavity, and endometrium.

Three-dimensional ultrasound (3D US) can be used to conduct $3 \mathrm{D}$ reconstructions of the uterine body, endometrial cavity, and endometrium, but it can only measure the volumes of these entities. In recent years, technological breakthroughs led to $3 \mathrm{D}$ reconstruction of the endometrial cavity (10); however, these $3 \mathrm{D}$ reconstructions yielded the simple measurements of length and width. Application of this technique required injection of saline, which can lead to patient distress. To our knowledge, taking advantage of two-dimensional ultrasound (2D US) images for $3 \mathrm{D}$ reconstructions have not previously been used to perform various measurements and analyses of the uterine body, endometrial cavity, and endometrium.

The main objectives of this study were as follows. Firstly, to employ medical software to implement 3D reconstructions of uterine body, endometrial cavity, and endometrium for the first time based on 2D US images and to carry out various measurements and analyses. Second, to measure routine parameters and determine differences in ECA, UBT, volume, and diameter lines of the uterine body between normal and arcuate uterus, between non-pregnant and pregnant infertile women, and between nulliparous and multiparous infertile women. Third, to identify differences in volume and thickness of the upper, middle, and lower regions of the endometrium in infertile women. Fourth, to explore the impact of age on these parameters. Finally, to provide a reference standard for using assisted reproductive technologies (ARTs), diagnosing uterine malformations, selecting intrauterine devices (IUDs), and implementing gynecological surgery. We present the following article in accordance with the STROBE reporting checklist (available at https://qims.amegroups.com/article/view/10.21037/ qims-21-812/rc).

\section{Methods}

\section{Study population}

This retrospective cross-sectional study included 204 patients, who visited The Second Hospital of Tianjin Medical University due to female infertility between June 2020 and May 2021. The patients were instructed to void urine before a $2 \mathrm{D}$ US standard examination. The examination was conducted by 2 ultrasonographers with extensive experience. Age, gravidity, and parity of the patients were obtained from the registration database. The exclusion criteria were as follows: uterine fibroids, adenomyosis, intrauterine adhesion, history of pelvic surgery, had not undergone a 2D US examination within 3-5 days after the end of their menstrual period, endometrial cavity had an IUD embedded, uterine malformation other than arcuate uterus and uterine body, or endometrial cavity and endometrium were impossible to identify on the 2D US image. Patients were screened independently and strictly by 2 researchers according to the inclusion and exclusion criteria. Both investigators had a thorough knowledge of gynecological diseases and extensive clinical experience. Any differences were resolved through discussion with a third researcher to achieve consensus. Ultimately, a total of 81 patients were included in the analysis. The flow diagram 


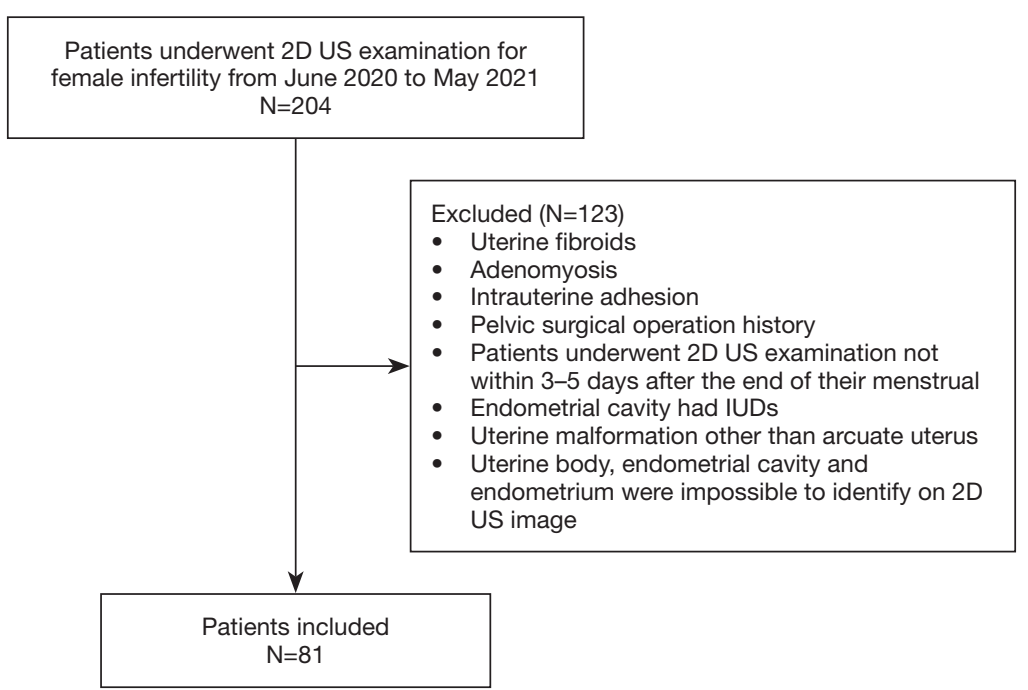

Figure 1 Flow diagram for patient inclusion and exclusion. 2D US, two-dimensional ultrasound; IUDs, intrauterine devices.

for patient inclusion and exclusion is presented in Figure 1. We defined the normal uterus based on the shape of the endometrial cavity. The shape of a normal uterus was similar to an inverted triangle (11). The arcuate uterus was defined as an indentation depth of $4-10 \mathrm{~mm}$ with an indentation angle $>90^{\circ}$ (12). Of the patients, 64 had a normal uterus, and ages ranged from 25 to 43 years (mean age $=32.64 \pm 4.72$ years). A total of 17 patients had an arcuate uterus, and their ages ranged from 23 to 42 years (mean age $=31.06 \pm 4.76$ years). The 64 patients with a normal uterus had the following gravidities and parities: gravidity $=0$ $(\mathrm{n}=31)$, gravidity $=1 \quad(\mathrm{n}=15)$, gravidity $>1 \quad(\mathrm{n}=18)$, parity $=0$ $(\mathrm{n}=48)$, and parity $=1(\mathrm{n}=16)$. This study was conducted in accordance with the Declaration of Helsinki (as revised in 2013) and approved by the Institutional Review Board (IRB) of The Second Hospital of Tianjin Medical University (No. KY2021K101), and individual consent for this retrospective analysis was waived.

\section{$3 D$ reconstruction}

The axial 2D US images with a slice thickness of $2.5 \mathrm{~mm}$ were imported into the Mimics software 19.0 (Materialize, Leuven, Belgium) in DICOM format. We created 3D models of the uterine body, endometrial cavity, and endometrium based on the 3D models' corresponding anatomical profiles. The reconstructed $3 \mathrm{D}$ models were then smoothed. Finally, these 3D models were imported for measurements and analyzed using 3-matic software 11.0
(Materialize, Leuven, Belgium). All 3D measurements and analyses were carried out by a professional researcher.

\section{Parameter measurements and analyses}

Uterine body volume (UBV), uterine body surface area (UBS), endometrial cavity volume (ECV), endometrial volume (EV), and endometrial surface area (ES) can be viewed through "Mimics software $\rightarrow$ properties". For the diameter line measurements, the highest and lowest point, the leftmost and rightmost point, and the most anterior and most posterior point of the uterine body, endometrial cavity, and endometrium were determined in the $\mathrm{Z}$-axis, $\mathrm{X}$-axis and Y-axis by 3-matic software, respectively. Uterine body length (UBL) and endometrial cavity length (ECL) were defined as the length from the highest to the lowest point measured on a corresponding 3D model. Uterine body width (UBW) and endometrial cavity width (ECW) were defined as the width from the leftmost to the rightmost point measured on a corresponding $3 \mathrm{D}$ model. Uterine body anteroposterior diameter (UBA) and ECA were defined as the distance from the most anterior to the most posterior point measured on a corresponding $3 \mathrm{D}$ model. Measurements of these distances were performed point to point using 3-matic software.

The steps for the measurement of the upper, middle, and lower regions of the endometrium were as follows. In the first step, the highest and lowest point of the endometrium was determined in the $\mathrm{Z}$-axis by 3 -matic software. The 

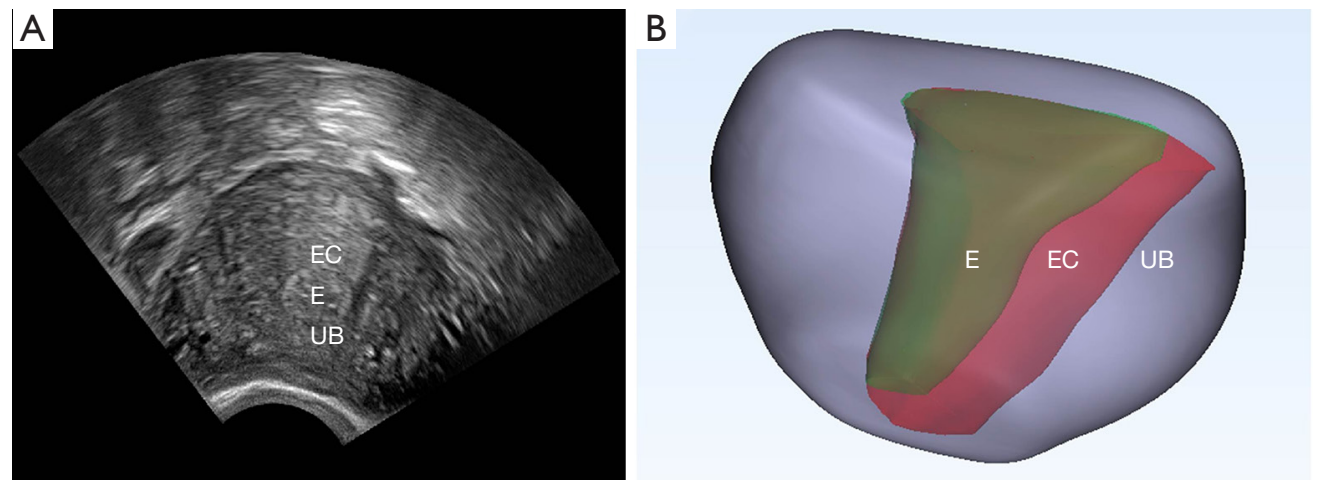

Figure 2 3D reconstructions of the UB, E, and EC. (A) Axial 2D US image. (B) 3D reconstructions of UB, E, and EC. 2D US, twodimensional ultrasound; 3D, three-dimensional; E, endometrium; EC, endometrial cavity; UB, uterine body.

endometrium length was defined first as the length from the highest to the lowest point of the endometrium. Then, the endometrial length was measured point to point using 3 -matic software. In the second step, 3 equal division points were determined using 3-matic software based on the endometrial length. In the third step, 3 horizontal planes were identified using 3 -matic software according to the 3 equal division points. In the last step, the endometrium was cut into 3 parts using the "plane cut" tool of 3-matic software. In this way, the reconstructed endometrium achieved equal-distance division.

The upper region of endometrial thickness (ETU), middle region of endometrial thickness (ETM), and lower region of endometrial thickness (ETL) were analyzed with 3-matic software. The upper region of endometrial volume (EVU), upper region of the endometrial surface area (ESU), middle region of the endometrial volume (EVM), middle region of the endometrial surface area (ESM), lower region of the endometrial volume (EVL), and the lower region of the endometrial surface area (ESL) were viewed using the "3-matic software -> properties" function. Similarly, the UBT and endometrial thickness (ET) were analyzed with 3-matic software.

\section{Statistical analysis}

Data were expressed as mean \pm standard deviation. MannWhitney $\mathrm{U}$ tests or 2 independent-samples $t$-tests were performed to compare differences between normal and arcuate uterus, between parity $=0$ and parity $=1$, and between the different regions of the endometrium. KruskallWallis tests or one-way analysis of variance (ANOVA) were used to detect differences between the different gravidities.
Correlations between age and parameters were analyzed using Pearson's or Spearman's correlation coefficients. Statistical tests were chosen depending on whether the data conformed to a normal distribution. All data analyses were performed using SPSS software 25.0 (IBM Corp., Armonk, NY, USA). A P value $<0.05$ was considered statistically significant.

\section{Results}

We successfully reconstructed 3D models of the uterine body, endometrial cavity, and endometrium from 2D US images with high accuracy using Mimics software (Figure 2). These models can be observed from different perspectives. The model-rendered morphology appeared highly realistic and enabled measurements and analyses of diameter lines, volume, surface area, and thickness (Figure 3). The reconstructed endometrium achieved equal-distance division (Figure 4). ECL, EVM, EVL, ESM, and ESL were significantly higher in the normal uterus than those in the arcuate uterus $(\mathrm{P}=0.000,0.001,0.006,0.000$, and 0.003, respectively). Age, gravidity, parity, UBL, UBW, UBA, UBT, UBV, UBS, ECW, ECA, ECV, EV, EVU, ES, ESU, ET, ETU, ETM, and ETL did not show significant differences between the 2 groups (Table 1).

With increasing gravidity, UBA, UBV, ECW, ECA, ES, ESU, and ET increased significantly $(\mathrm{P}=0.035,0.027,0.010$, $0.048,0.039,0.010$, and 0.036 , respectively). No significant differences were observed in age, UBL, UBW, UBT, UBS, EVL, ECV, EV, EVU, EVM, EVL, ESM, ESL, ETU, ETM, and ETL among the 3 groups (Table 2).

Age, UBW, UBV, UBS, ECW, ECV, EV, EVU, EVM, EVL, ES, ESU, ESM, ESL, ET, and ETU in the parity 

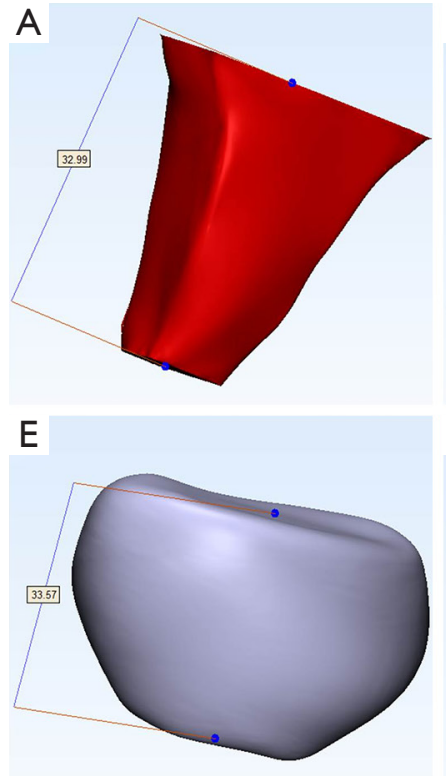

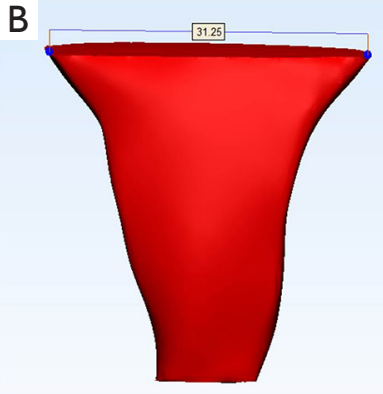

$\mathrm{F}$

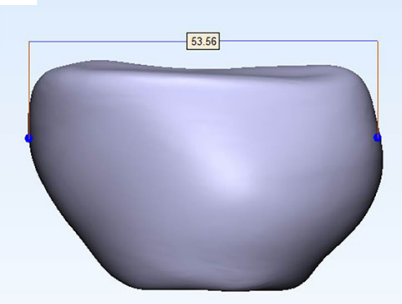

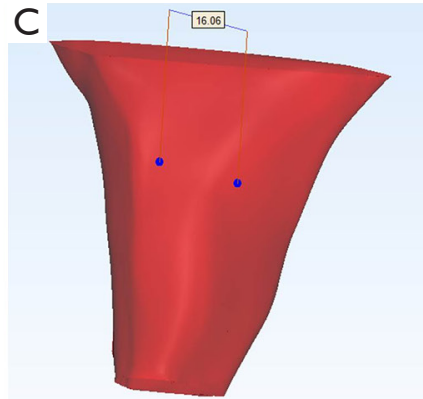
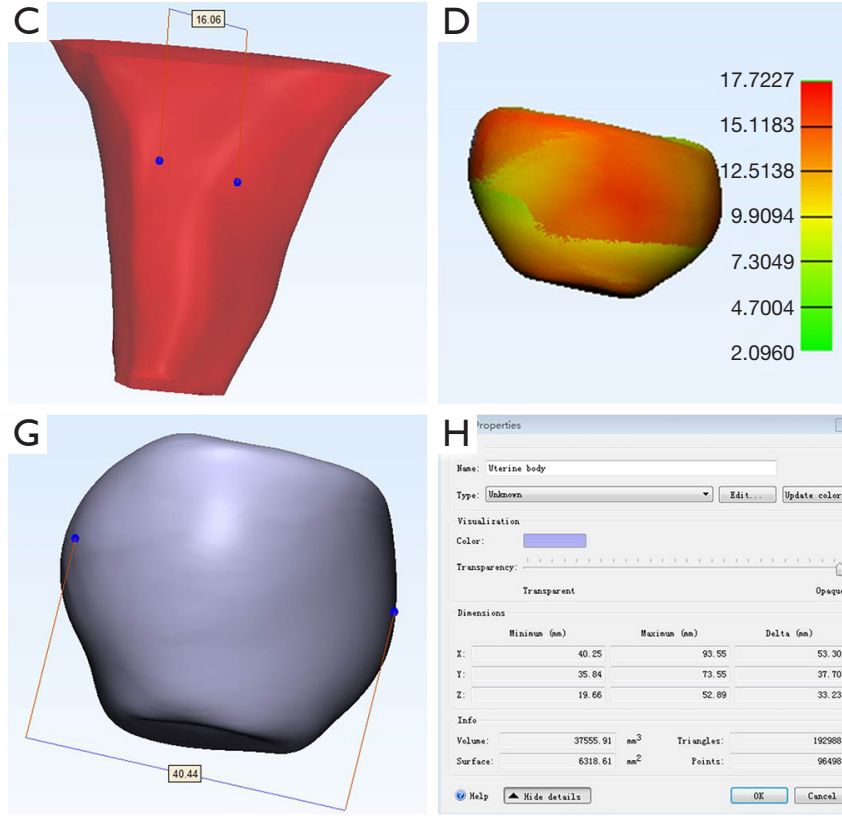

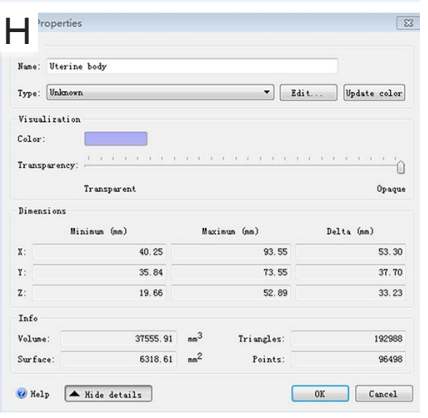

Figure 3 3D analyses of length $(\mathrm{mm})$, width $(\mathrm{mm})$, anteroposterior diameter $(\mathrm{mm})$, thickness $(\mathrm{mm})$, volume $\left(\mathrm{mm}^{3}\right)$, and surface area $\left(\mathrm{mm}^{2}\right)$. (A) The highest and lowest point of the endometrial cavity was determined in the Z-axis by 3-matic software. The ECL was measured from the highest to the lowest point. The ECL was $32.99 \mathrm{~mm}$. (B) the leftmost and rightmost point of endometrial cavity was determined in the $\mathrm{X}$-axis by 3-matic software. The ECW was measured from the leftmost to the rightmost point. The ECW was $31.25 \mathrm{~mm}$. (C) The most anterior and most posterior point of the endometrial cavity was determined in the Y-axis by 3-matic software. The ECA was measured from the most anterior to the most posterior point. The ECA was $16.05 \mathrm{~mm}$. (D) The UBT was analyzed using 3-matic software. The UBT was $17.72 \mathrm{~mm}$. (E) The highest and lowest point of the uterine body was determined in the Z-axis by 3 -matic software. The UBL was measured from the highest to the lowest point. The UBL was $33.57 \mathrm{~mm}$. (F) The leftmost and the rightmost point of the uterine body was determined in the $\mathrm{X}$-axis by 3-matic software. The UBW was measured from the leftmost to the rightmost point. The UBW was $53.56 \mathrm{~mm}$. (G) The most anterior and most posterior point of uterine body was determined in the Y-axis by 3-matic software. The UBA was measured from the most anterior to the most posterior point. The UBA was $40.44 \mathrm{~mm}$. (H) Schematic diagram of the properties of the uterine body. The UBV was $37,555.91 \mathrm{~mm}^{3}$. The UBS was $6,318.61 \mathrm{~mm}^{2} .3 \mathrm{D}$, three-dimensional; ECA, endometrial cavity anterior-posterior diameter; ECL, endometrial cavity length; ECW, endometrial cavity width; UBA, uterine body anterior-posterior diameter; UBL, uterine body length; UBS, uterine body surface area; UBT, uterine body thickness; UBV, uterine body volume; UBW, uterine body width.

1 infertile women were significantly greater than those in the parity 0 infertile women $(\mathrm{P}=0.015,0.018,0.004,0.024$, $0.002,0.002,0.004,0.003,0.016,0.019,0.001,0.013$, $0.007,0.013,0.039$, and 0.010 , respectively). There were no significant differences in UBL, UBA, UBT, ECL, ECA, ETM, and ETL (Table 3).

Both EVU and EVM were significantly greater than EVL (both $\mathrm{P}=0.000$ ). Both ETU and ETM were significantly greater than ETL $(P=0.009$ and 0.001 , respectively). The ESU was significantly greater than both $\mathrm{ESM}$ and ESL (both $\mathrm{P}=0.000$ ). The ESM was significantly greater than ESL $(\mathrm{P}=0.000)$. Values were not significantly different between EVU and EVM and between ETU and ETM (Table 4).
Correlation analysis revealed that age correlated positively with UBL $(\mathrm{r}=0.248, \mathrm{P}=0.048)$, UBW $(\mathrm{r}=0.293$, $\mathrm{P}=0.019), \mathrm{UBA}(\mathrm{r}=0.263, \mathrm{P}=0.036), \mathrm{UBV}(\mathrm{r}=0.365, \mathrm{P}=0.003)$, UBS $(r=0.332, \mathrm{P}=0.007), \mathrm{ECL}(\mathrm{r}=0.277, \mathrm{P}=0.027), \mathrm{ECV}$ $(\mathrm{r}=0.306, \mathrm{P}=0.014), \mathrm{EVU}(\mathrm{r}=0.274, \mathrm{P}=0.028)$, ES $(\mathrm{r}=0.278$, $\mathrm{P}=0.026)$, and $\mathrm{ESU}(\mathrm{r}=0.311, \mathrm{P}=0.012)$. No significant correlations were found between age and UBT, ECW, ECA, EV, EVM, EVL, ESM, ESL, ET, ETU, ETM, and ETL (Table 5).

\section{Discussion}

In gynecological examinations, US is the most commonly used imaging method. In a study by Zhao et al., an 

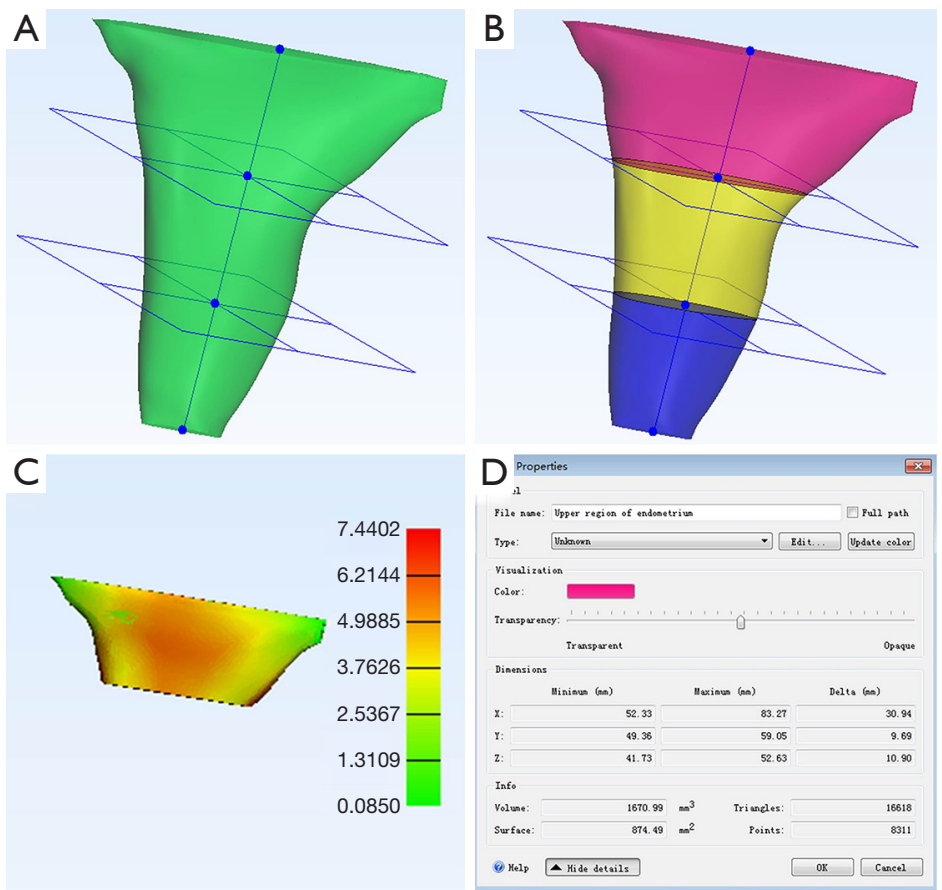

Figure 4 Equal-distance division of the endometrium. (A) The highest and lowest point of the endometrium was determined in the Z-axis by 3-maic software. The endometrial length was defined first as the length from the highest to the lowest point of the endometrium. Then, 3 equal division points were determined using 3-matic software according to the endometrial length. (B) Three horizontal planes were identified first using 3-matic software based on the 3 equal division points. Then, the endometrium was cut into 3 parts using the "plane cut" tool of the 3-matic software. (C) The ETU was analyzed with 3-matic software. The ETU was 7.44 mm. (D) Schematic diagram of the properties of the upper region of the endometrium. The EVU is $1,670.99 \mathrm{~mm}^{3}$. The ESU is $874.49 \mathrm{~mm}^{2}$. ETU, upper region of endometrial thickness; EVU, upper region of endometrial volume; ESU, upper region of endometrial surface area.

accurate 3D model of the malleus and incus was developed with computed tomography data by means of Mimics software (13). For the first time, this study employed the use of Mimics software to implement 3D reconstructions of the uterine body, endometrial cavity, and endometrium based on 2D US images and resulted in various measurements and analyses. Our study extends the current knowledge about infertile women and arcuate uterus, highlighting the nuances of morphology of the uterine body, endometrial cavity, and endometrium. The findings from this study have important implication for ARTs, the diagnosis of uterine malformations, selecting IUDs, and performing gynecological surgery.

No consensus has been reached about the diagnostic criteria of uterine malformations. The definitions of normal and/or arcuate uterus by the European Society of Human Reproduction and Embryology, the European Society for Gynaecological Endoscopy (ESHRE-ESGE), and the American Society for Reproductive Medicine (ASRM) classification systems for congenital uterine anomalies remain controversial (14). Several studies have shown that a normal/arcuate uterus can easily be misdiagnosed as septate uterus using the standard ESHRE-ESGE criteria $(15,16)$. Although the accuracy of the ASRM classification system was higher than the accuracy of the ESHRE-ESGE classification system for diagnosing septate uterus, the diagnosis of septate uterus is prone to error (17). None of the ASRM, ESHREESGE, or Congenital Uterine Malformation by Experts (CUME) has provided detailed descriptions of normal and arcuate uterus. The 3D morphological parameters can diagnose the thoracic myelopathy resulting from thoracic ossification of the ligamentum flavum more accurately than 2D morphological parameters (18). This study found that ECL, EVM, EVL, ESM, and ESL were significantly lower in arcuate uterus compared to normal uterus using the $3 \mathrm{D}$ measurement approach. Our results may provide an explanation for previous research findings. In Sun et al.'s study, infertile women with normal/arcuate uterus 
Table 1 Comparison of normal and arcuate uterine parameters (mean \pm SD)

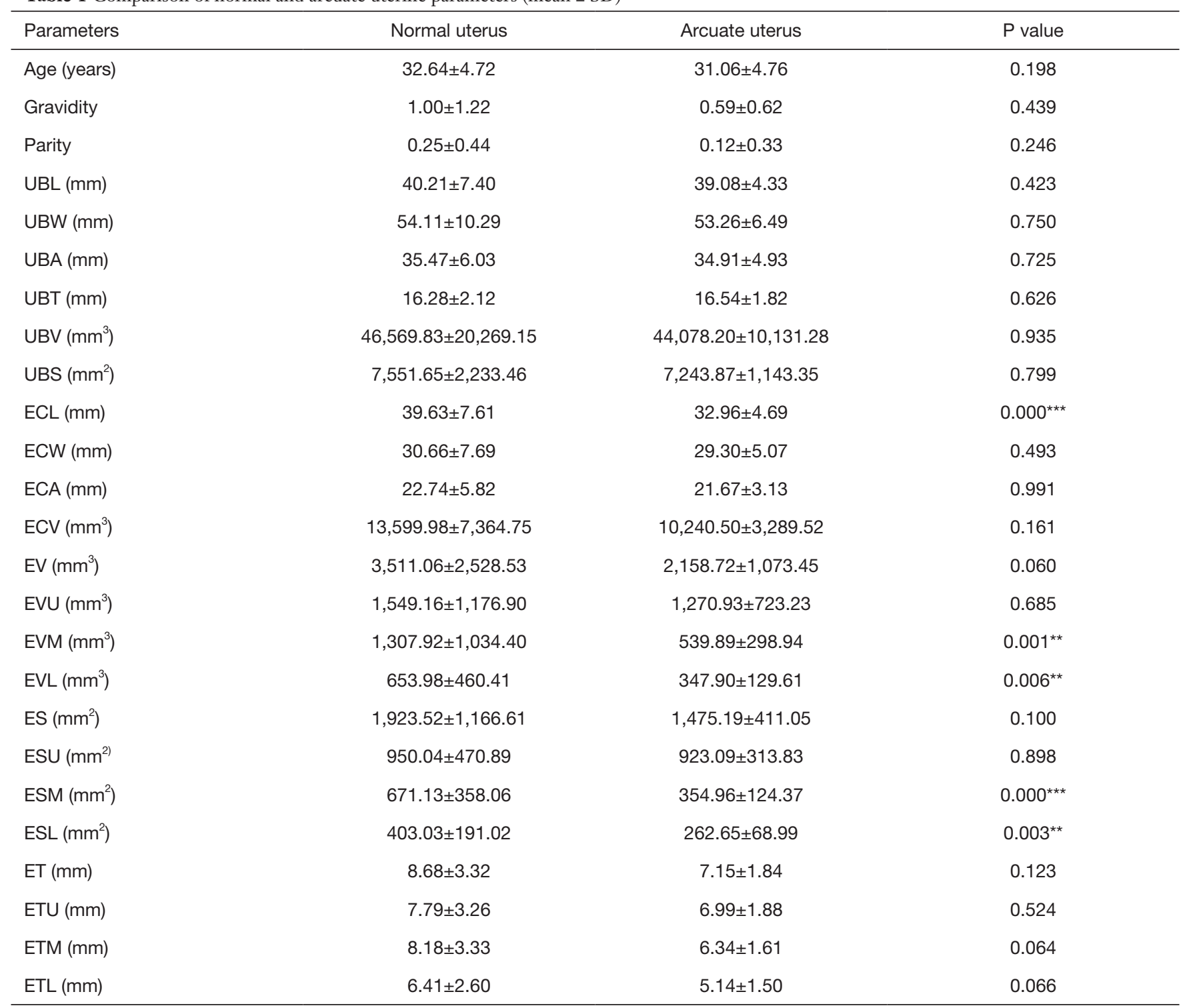

${ }^{* \star}, \mathrm{P}<0.01 ;{ }^{* \star *}, \mathrm{P}<0.001$. SD, standard deviation; UBL, uterine body length; UBW, uterine body width; UBA, uterine body anteroposterior diameter; UBT, uterine body thickness; UBV, uterine body volume; UBS, uterine body surface area; ECL, endometrial cavity length; ECW, endometrial cavity width; ECA, endometrial cavity anteroposterior diameter; ECV, endometrial cavity volume; EV, endometrial volume; EVU, upper region of endometrial volume; EVM, middle region of endometrial volume; EVL, lower region of endometrial volume; ES, endometrial surface area; ESU, upper region of endometrial surface area; ESM, middle region of endometrial surface area; ESL, lower region of endometrial surface area; ET, endometrial thickness; ETU, upper region of endometrial thickness; ETM, middle region of endometrial thickness; ETL, lower region of endometrial thickness.

underwent embryo transplantation. They discovered that the conception and implantation rates of arcuate uterus were significantly lower than those of normal uterus (11). This may be because EVM, EVL, ESM, and ESL of arcuate uterus are significantly lower than those of normal uterus. Decreases in EV and ES are accompanied by corresponding decreases in nutrition, blood supply, and space available for gestation.

The ECW and ECL are vital reference standards for selecting IUDs and implementing gynecological operations (19). Wildemeersch et al. found that the width of a conventional IUD $(32 \mathrm{~mm})$ was significantly greater 
Table 2 Comparison of different gravidity parameters (mean \pm SD)

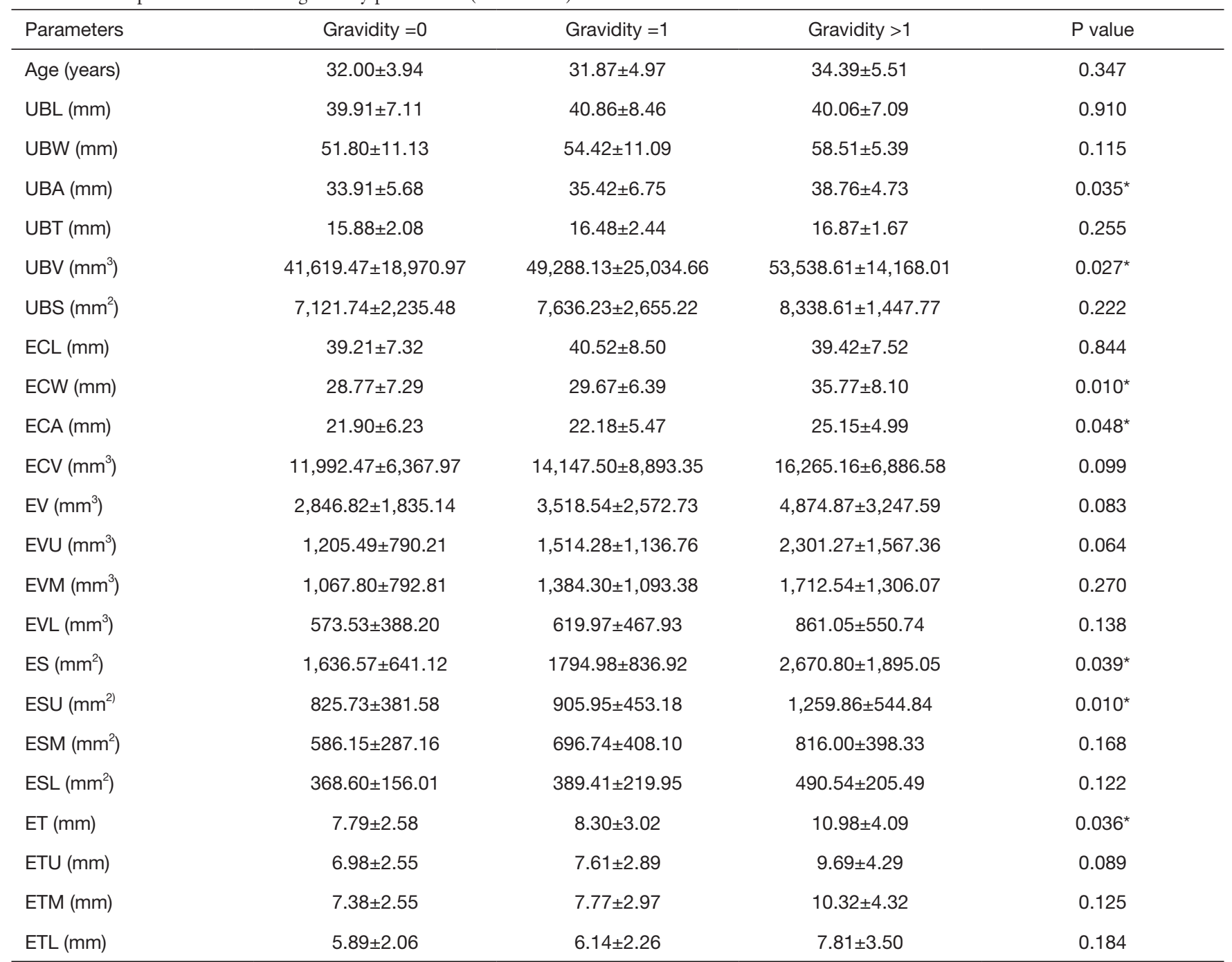

*, P<0.05. SD, standard deviation; UBL, uterine body length; UBW, uterine body width; UBA, uterine body anteroposterior diameter; UBT, uterine body thickness; UBV, uterine body volume; UBS, uterine body surface area; ECL, endometrial cavity length; ECW, endometrial cavity width; ECA, endometrial cavity anteroposterior diameter; ECV, endometrial cavity volume; EV, endometrial volume; EVU, upper region of endometrial volume; EVM, middle region of endometrial volume; EVL, lower region of endometrial volume; ES, endometrial surface area; ESU, upper region of endometrial surface area; ESM, middle region of endometrial surface area; ESL, lower region of endometrial surface area; ET, endometrial thickness; ETU, upper region of endometrial thickness; ETM, middle region of endometrial thickness; ETL, lower region of endometrial thickness.

than the mean width of the endometrial cavity (20). This mismatch causes pain and bleeding, and has even been assumed to contribute to the contraceptive failure. However, there is still controversy concerning the results of the mean ECW. The mean ECW of nulliparous infertile women in Wildemeersch et al.'s and Benacerraf et al.'s studies were 21.6 and $27.2 \mathrm{~mm}$, respectively $(20,21)$, whereas Goldstuck's literature review concluded that the mean ECW of nulliparous women was $28.2 \mathrm{~mm}$ according to imaging measurements (19). Similarly, this study found that the mean ECW of nulliparous women was less than the width of conventional IUDs and increased significantly with gravidity and parity, which is in agreement with Benacerraf et al.'s study (21). The mean ECW value of the current study was $28.98 \mathrm{~mm}$.

These discrepancies could be due to the inaccurate diameter line measurements provided by US. The endometrial cavity is not always a regular ellipsoid shape, 
Table 3 Comparison of different parity parameters (mean \pm SD)

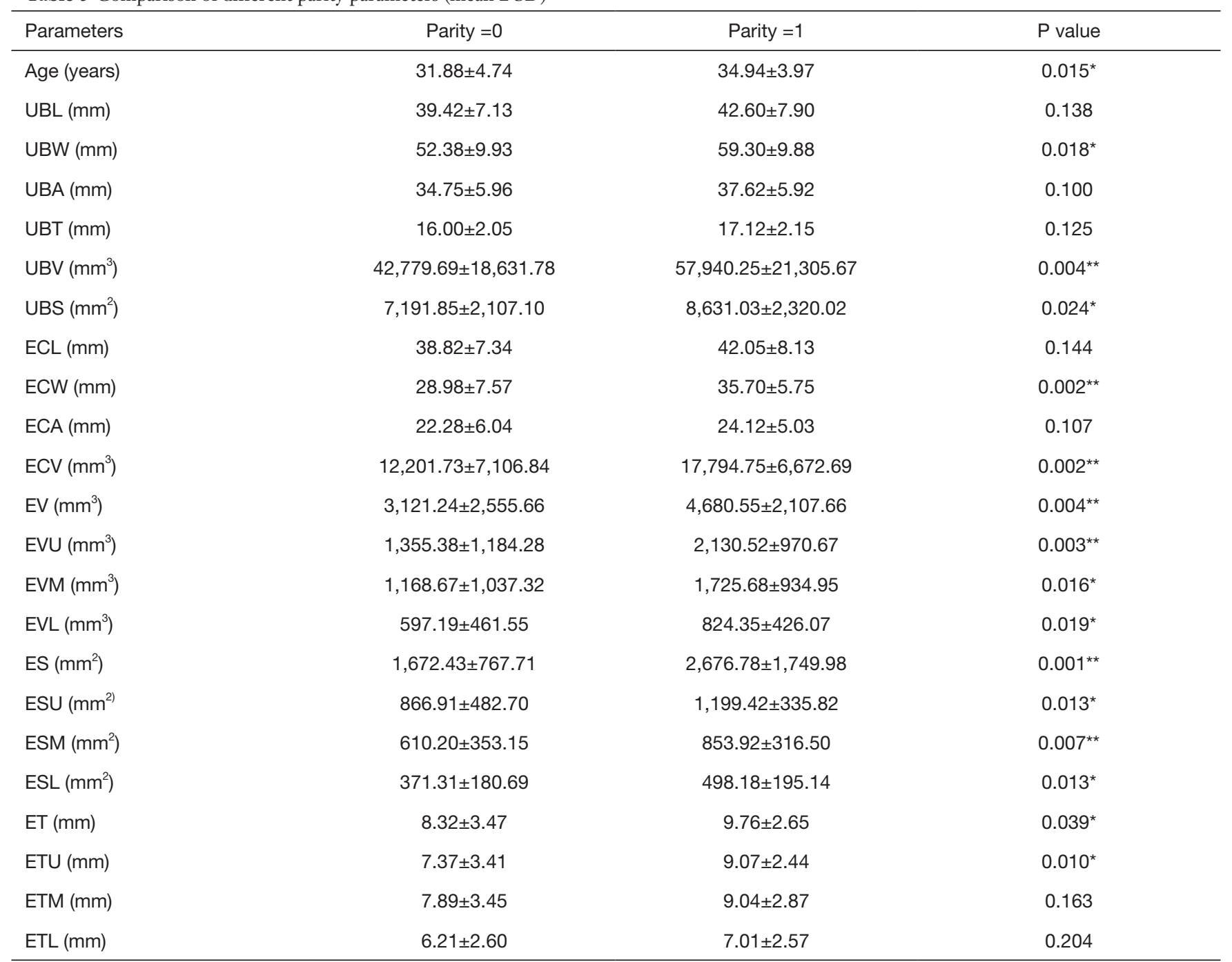

*, $\mathrm{P}<0.05 ;{ }^{*}, \mathrm{P}<0.01 . \mathrm{SD}$, standard deviation; UBL, uterine body length; UBW, uterine body width; UBA, uterine body anteroposterior diameter; UBT, uterine body thickness; UBV, uterine body volume; UBS, uterine body surface area; ECL, endometrial cavity length; ECW, endometrial cavity width; ECA, endometrial cavity anteroposterior diameter; ECV, endometrial cavity volume; EV, endometrial volume; EVU, upper region of endometrial volume; EVM, middle region of endometrial volume; EVL, lower region of endometrial volume; ES, endometrial surface area; ESU, upper region of endometrial surface area; ESM, middle region of endometrial surface area; ESL, lower region of endometrial surface area; ET, endometrial thickness; ETU, upper region of endometrial thickness; ETM, middle region of endometrial thickness; ETL, lower region of endometrial thickness.

and, in particular, moderate distortion of the triangular aspect (22) can influence the accuracy of measurement. The ECW is often subjectively determined, which influences the end results. The 3-matic software used in our study was able to automatically analyze the ECW, which guaranteed the accuracy of ECW measurement.

This study showed that the mean ECL measured in women of different parities was larger than the length of the conventional IUDs, which is consistent with the findings of Canteiro et al. (23). Both studies also found that there is no significant difference in mean ECL between women of different parities. In this study, no significant differences in mean ECL were found between women of different gravidities. Our results showed that the mean ECW was less than the width of conventional IUDs, while the mean ECL was greater than that of conventional IUDs. The size of the endometrial cavity can be evaluated using precise 3D measurement and analysis prior to inserting IUDs. Suitable 
Table 4 Comparison of parameters related to different regions of endometrium in normal uterus

\begin{tabular}{lcc}
\hline Parameters & Value, mean \pm SD & P value \\
\hline EVU vs. EVM $\left(\mathrm{mm}^{3}\right)$ & $1,549.16 \pm 1,176.90$ vs. $1,307.92 \pm 1,034.40$ & 0.217 \\
EVU vs. EVL $\left(\mathrm{mm}^{3}\right)$ & $1,549.16 \pm 1,176.90$ vs. $653.98 \pm 460.41$ & $0.000^{\star \star \star}$ \\
EVM vs. EVL $\left(\mathrm{mm}^{3}\right)$ & $1,307.92 \pm 1,034.40$ vs. $653.98 \pm 460.41$ & $0.000^{\star \star \star}$ \\
ESU vs. ESM $\left(\mathrm{mm}^{2}\right)$ & $950.04 \pm 470.89$ vs. $671.13 \pm 358.06$ & $0.000^{\star \star \star}$ \\
ESU vs. ESL $\left(\mathrm{mm}^{2}\right)$ & $950.04 \pm 470.89$ vs. $403.03 \pm 191.02$ & $0.000^{\star \star \star}$ \\
ESM vs. ESL $\left(\mathrm{mm}^{2}\right)$ & $671.13 \pm 358.06$ vs. $403.03 \pm 191.02$ & $0.000^{\star \star \star}$ \\
ETU vs. ETM $\left(\mathrm{mm}^{2}\right)$ & $7.79 \pm 3.26$ vs. $8.18 \pm 3.33$ & 0.548 \\
ETU vs. ETL $(\mathrm{mm})$ & $7.79 \pm 3.26$ vs. $6.41 \pm 2.60$ & $0.009^{\star \star}$ \\
ETM vs. ETL $(\mathrm{mm})$ & $8.18 \pm 3.33$ vs. $6.41 \pm 2.60$ & $0.001^{\star \star}$ \\
\hline
\end{tabular}

${ }^{* *}, \mathrm{P}<0.01$; ${ }^{* *}, \mathrm{P}<0.001 . \mathrm{SD}$, standard deviation; EVU, upper region of endometrial volume; EVM, middle region of endometrial volume; $\mathrm{EVL}$, lower region of endometrial volume; ESU, upper region of endometrium surface area; ESM, middle region of endometrium surface area; ESL, lower region of endometrial surface area; ETU, upper region of endometrial thickness; ETM, middle region of endometrial thickness; ETL, lower region of endometrial thickness.

IUDs can also be selected according to width of the endometrial cavity. In this way, the negative effects of IUDs can be reduced.

Currently, research on the ECA is scarce. Goldstuck obtained the ECV using 3D US and then studied the ECA (range, 6.29 to $38.2 \mathrm{~mm}$ ), which was derived from the ellipsoid formula (6). As stated above, the endometrial cavity does not exhibit a regular ellipsoid shape. Therefore, results of ECA measurement using this formula cannot be accurate. In our study, the analysis of ECA was automatically performed on the $3 \mathrm{D}$ model of the endometrial cavity. Using this precise approach, we found that the mean ECA of nulliparous women was $22.28 \mathrm{~mm}$ and increased dramatically with gravidity. The ECV was also obtained using automatic analysis. In our study, the ECV increased significantly with parity but not with gravidity. This suggests that the ECV is primarily affected by parity.

The shape of the uterus is also not regular. Gao et al. found that with increasing age, the shape of the uterus gradually develops into a nearly circular shape in women with primary infertility (9). Verguts et al. reported that the ratio of the length of the uterus to the width of the uterus was close to the golden ratio in a 20 -year-old female (24). Likewise, the limitations of using the ellipsoid formula to calculate the volume of the uterus also apply. In our study, automatic analysis of UBV was performed with 3-matic software to avoid personal subjective bias. This study showed that UBV increased significantly with the increase in gravidity and parity. In this study, UBA increased significantly with increasing gravidity. There were no differences in UBT between different gravidity and parity groups. The UBT did not vary with gravity and parity. Moreover, UBW and UBS significantly increased with increasing parity. Our study fills the research gap on the impact of gravidity and parity on the uterine body.

Consistent with Benacerraf et al.'s study (21), our results indicated that the ET increased significantly with gravidity and parity; however, our study demonstrated this point more powerfully. The study by Benacerraf $e t a l$. did not control patients' menstrual cycle phases, whereas we selected patients who underwent 2D US standard examination within 3-5 days after the end of their menstrual period. Another reason for inaccurate ET measurement was that the ET measurement in a $2 \mathrm{D}$ plane was required to determine the widest distance on the cut surface of the endometrium. However, the endometrium has irregular contours, which may increase subjective errors. The 3-matic software provided accurate measurement for the ET. This study also revealed the effects of gravidity and parity on the upper, middle, and lower region of the endometrium. On the one hand, ES and ESU increased significantly with increasing gravidity. On the other hand, parity had no impact on ETM and ETL.

The endometrium provides nutrition and blood support to the endometrial cavity and is regulated by estrogen and progestogen. The endometrium plays a pivotal role in the 
Table 5 Correlations between age and parameters relating to the normal uterus

\begin{tabular}{|c|c|c|}
\hline Parameters & r value & $P$ value \\
\hline UBL (mm) & 0.248 & $0.048^{*}$ \\
\hline UBW (mm) & 0.293 & $0.019^{*}$ \\
\hline UBA (mm) & 0.263 & $0.036^{\star}$ \\
\hline UBT (mm) & 0.193 & 0.127 \\
\hline $\mathrm{UBV}\left(\mathrm{mm}^{3}\right)$ & 0.365 & $0.003^{* *}$ \\
\hline UBS $\left(\mathrm{mm}^{2}\right)$ & 0.332 & $0.007^{\star *}$ \\
\hline $\mathrm{ECL}(\mathrm{mm})$ & 0.277 & $0.027^{\star}$ \\
\hline ECW (mm) & 0.209 & 0.097 \\
\hline $\mathrm{ECA}(\mathrm{mm})$ & 0.178 & 0.159 \\
\hline $\mathrm{ECV}\left(\mathrm{mm}^{3}\right)$ & 0.306 & $0.014^{*}$ \\
\hline $\mathrm{EV}\left(\mathrm{mm}^{3}\right)$ & 0.228 & 0.070 \\
\hline $\mathrm{EVU}\left(\mathrm{mm}^{3}\right)$ & 0.274 & $0.028^{*}$ \\
\hline $\mathrm{EVM}\left(\mathrm{mm}^{3}\right)$ & 0.205 & 0.104 \\
\hline $\mathrm{EVL}\left(\mathrm{mm}^{3}\right)$ & 0.105 & 0.407 \\
\hline $\mathrm{ES}\left(\mathrm{mm}^{2}\right)$ & 0.278 & $0.026^{*}$ \\
\hline $\mathrm{ESU}\left(\mathrm{mm}^{2}\right)$ & 0.311 & $0.012^{*}$ \\
\hline $\mathrm{ESM}\left(\mathrm{mm}^{2}\right)$ & 0.214 & 0.090 \\
\hline $\mathrm{ESL}\left(\mathrm{mm}^{2}\right)$ & 0.105 & 0.407 \\
\hline $\mathrm{ET}(\mathrm{mm})$ & 0.089 & 0.485 \\
\hline ETU (mm) & 0.233 & 0.063 \\
\hline ETM (mm) & 0.077 & 0.547 \\
\hline ETL (mm) & -0.017 & 0.895 \\
\hline
\end{tabular}

${ }^{*}, \mathrm{P}<0.05 ;{ }^{* *}, \mathrm{P}<0.01$. UBL, uterine body length; UBW, uterine body width; UBA, uterine body anteroposterior diameter; UBT, uterine body thickness; UBV, uterine body volume; UBS, uterine body surface area; ECL, endometrial cavity length; ECW, endometrial cavity width; ECA, endometrial cavity anteroposterior diameter; ECV, endometrial cavity volume; EV, endometrial volume; EVU, upper region of endometrial volume; EVM, middle region of endometrial volume; EVL, lower region of endometrial volume; ES, endometrial surface area; ESU, upper region of endometrial surface area; ESM, middle region of endometrial surface area; ESL, lower region of endometrial surface area; ET, endometrial thickness; ETU, upper region of endometrial thickness; ETM, middle region of endometrial thickness; ETL, lower region of endometrial thickness. conception and development of the embryo $(25,26)$. The normal ET value of the proliferative endometrial phase in 0 - to 20 -year-old females is $4.5 \pm 2.8 \mathrm{~mm}$ (27). Our results showed that the ET in nulliparous infertile women with a normal uterus was $8.32 \pm 3.47 \mathrm{~mm}$, which is greater than that of the 0- to 20-year-old females. Moreover, there were notable discrepancies between the upper, middle, and lower regions of the endometrium in infertile women. This study demonstrated that the volume, thickness, and surface area of the upper and middle regions of the endometrium were significantly greater than those in the lower region of the endometrium.

Gao et al. reported that the diameter lines of the uterus were significantly and positively correlated with age (9). This study suggested that UBL, UBW, UBA, UBV, UBS, ECL, and ECV correlated significantly with age. This illustrated that the whole shape of the uterus changes with aging as well as the related parameters of the uterine body and the endometrial cavity. In Gao et al.'s study, the optimal diameter lines and optimal volume of the uterus range for infertile women who had a higher chance of ART treatment success were outlined (28). Gao et al.'s study involved Southern Chinese women, while our study focused on Northern Chinese women. Our study and Gao et al.'s study provided important reference data for follow-up research relating to the uterus and its related structures in Chinese women. No correlation between UBT and age was observed. The EVU, ESU, and ES increased significantly with increasing age. No correlations were found between other parameters of endometrium and age. Age was shown to mainly affect the upper region of the endometrium.

This study had the following limitations. First, although this paper provided a new method for accurate measurement and analysis of the uterine body, endometrial cavity, and endometrium, it requires manual selection of the region of interest according to the corresponding anatomical profiles. Therefore, this approach is time-consuming. Future research should explore automatic or semi-automatic approaches of selecting the region of interest. Second, our study population involved participants with parity $=0$ and parity $=1$. We did not compare the discrepancy in morphological parameters between parity $>1$ and parity $\leq 1$. Third, only one professional researcher conducted 
all 3D measurements and analyses, and interobserver variability was not assessed. However, this professional researcher had specialist anatomical knowledge and received professional training in the use of medical software. The researcher was able to position the uterus and its related structure accurately in 2D US images. In our study, the morphometric parameters such as volume, thickness, and surface area were automatically analyzed using medical software. This approach largely avoided subjective effects. Fourth, the present study was limited by its retrospective data collection. Finally, the number of samples was relatively small. Future studies will need a larger sample to confirm our results. However, our precise approach can be used to explore the more variable characteristics of gynecological diseases and provides reference criteria for the diagnosis and treatment of these diseases.

\section{Conclusions}

This study provides reference data for volume and thickness of the endometrium in the different anatomical regions of normal and arcuate uterus. Age mainly affects the upper region of endometrium. The UBT does not vary with gravity, parity, or age. The $3 \mathrm{D}$ measurement provides a precise way to quantify the morphological parameters of gynecological diseases.

\section{Acknowledgments}

Funding: This work was supported by grants from the National Natural Science Foundation of China (No. 31871212, Lidong Zhai), Postdoctoral Science Foundation of China (No. 2016M601272, Lidong Zhai).

\section{Footnote}

Reporting Checklist: The authors have completed the STROBE reporting checklist. Available at https://qims. amegroups.com/article/view/10.21037/qims-21-812/rc

Conflicts of Interest: All authors have completed the ICMJE uniform disclosure form (available at https://qims. amegroups.com/article/view/10.21037/qims-21-812/coif). The authors have no conflicts of interest to declare.

Ethical Statement: The authors are accountable for all aspects of the work in ensuring that questions related to the accuracy or integrity of any part of the work are appropriately investigated and resolved. The study was conducted in accordance with the Declaration of Helsinki (as revised in 2013). The study was approved by the Institutional Review Board (IRB) of The Second Hospital of Tianjin Medical University (No. KY2021K101), and individual consent for this retrospective analysis was waived.

Open Access Statement: This is an Open Access article distributed in accordance with the Creative Commons Attribution-NonCommercial-NoDerivs 4.0 International License (CC BY-NC-ND 4.0), which permits the noncommercial replication and distribution of the article with the strict proviso that no changes or edits are made and the original work is properly cited (including links to both the formal publication through the relevant DOI and the license). See: https://creativecommons.org/licenses/by-nc-nd/4.0/.

\section{References}

1. Li J, Wang Y, Tang R, Peng Y, Wang Y, Liu B, Jiang Y, Liu G, Lin S, Chen R. Changes in ultrasound uterine morphology and endometrial thickness during ovarian aging and possible associated factors: findings from a prospective study. Menopause 2020;27:794-800.

2. Bardin R, Ashwal E, Zilber H, Tenenbaum-Gavish K, Hiersch L, Hadar E, Meizner I, Gabbay-Benziv R. Sonographic appearance of the uterus in the early puerperium in vaginal versus cesarean deliveries: a prospective study. J Matern Fetal Neonatal Med 2018;31:1983-8.

3. Liang H, Li L, Yuan W, Zou Y, Gao ES, Duffy JM, Wu SC. Dimensions of the endometrial cavity and intrauterine device expulsion or removal for displacement: a nested case-control study. BJOG 2014;121:997-1004.

4. Çintesun FNI, Çintesun E, Esenkaya Ü, Günenc O. Uterine dimensions and intrauterine device malposition: can ultrasound predict displacement or expulsion before it happens? Arch Gynecol Obstet 2020;302:1181-7.

5. Aouad P, Serhal A, Dion E, Buy JN. Documentation of normal shape and measurements of the uterine fundus at reproductive age. Acta Radiol 2021;62:667-72.

6. Goldstuck ND. Dimensional analysis of the endometrial cavity: how many dimensions should the ideal intrauterine device or system have? Int J Womens Health 2018;10:165-8.

7. Yuan X, Saravelos SH, Wang Q, Xu Y, Li TC, Zhou C. Endometrial thickness as a predictor of pregnancy outcomes in 10787 fresh IVF-ICSI cycles. Reprod Biomed 
Online 2016;33:197-205.

8. Steiner N, Ates S, Shaulov T, Tannus S, Dahan MH. Effect of uterine dimensions on live birth rates after single embryo transfer in infertile women. Reprod Biomed Online 2020;41:663-70.

9. Gao H, Liu DE, Li Y, Tang J, Hu S, Wu X, Tian Z, Tan H. Uterine dimensions in gravida 0 phase according to age, body mass index, and height in Chinese infertile women. Medicine (Baltimore) 2018;97:e12068.

10. Ludwin A, Martins WP, Ludwin I. Uterine cavity imaging, volume estimation and quantification of degree of deformity using automatic volume calculation: description of technique. Ultrasound Obstet Gynecol 2017;50:138-40.

11. Sun Y, Fang L, Su Y, Guo Y. Uterine cavity shape and the best site for embryo transfer. Int J Gynaecol Obstet 2009; 105:140-4.

12. Surrey ES, Katz-Jaffe M, Surrey RL, Small AS, Gustofson RL, Schoolcraft WB. Arcuate uterus: is there an impact on in vitro fertilization outcomes after euploid embryo transfer? Fertil Steril 2018;109:638-43.

13. Zhao D, Lu Q, Zou S, Sun J, Hu F. Accuracy of individualized 3D modeling of ossicles using highresolution computed tomography imaging data. Quant Imaging Med Surg 2021;11:2406-14.

14. Ludwin A, Ludwin I. Comparison of the ESHRE-ESGE and ASRM classifications of Müllerian duct anomalies in everyday practice. Hum Reprod 2015;30:569-80.

15. Ludwin A, Ludwin I, Kudla M, Kottner J. Reliability of the European Society of Human Reproduction and Embryology/European Society for Gynaecological Endoscopy and American Society for Reproductive Medicine classification systems for congenital uterine anomalies detected using three-dimensional ultrasonography. Fertil Steril 2015;104:688-97.e8.

16. Ludwin A, Ludwin I, Coelho Neto MA, Nastri CO, Bhagavath B, Lindheim SR, Martins WP. Septate uterus according to ESHRE/ESGE, ASRM and CUME definitions: association with infertility and miscarriage, cost and warnings for women and healthcare systems. Ultrasound Obstet Gynecol 2019;54:800-14.

17. Ludwin A, Martins WP, Nastri CO, Ludwin I, Coelho Neto MA, Leitão VM, Acién M, Alcazar JL, Benacerraf B, Condous G, De Wilde RL, Emanuel MH, Gibbons W, Guerriero S, Hurd WW, Levine D, Lindheim S, Pellicer A, Petraglia F, Saridogan E. Congenital Uterine Malformation by Experts (CUME): better criteria for distinguishing between normal/arcuate and septate uterus?
Ultrasound Obstet Gynecol 2018;51:101-9.

18. Yan C, Tan HY, Ji CL, Yu XW, Jia HC, Li FD, Jiang GC, Li WS, Zhou FF, Ye Z, Sun JC, Shi JG. The clinical value of three-dimensional measurement in the diagnosis of thoracic myelopathy caused by ossification of the ligamentum flavum. Quant Imaging Med Surg 2021;11:2040-51.

19. Goldstuck N. Assessment of uterine cavity size and shape: a systematic review addressing relevance to intrauterine procedures and events. Afr J Reprod Health 2012;16:130-9.

20. Wildemeersch D, Hasskamp T, Nolte K, Jandi S, Pett A, Linden S, van Santen M, Julen O. A multicenter study assessing uterine cavity width in over 400 nulliparous women seeking IUD insertion using 2D and 3D sonography. Eur J Obstet Gynecol Reprod Biol 2016;206:232-8.

21. Benacerraf BR, Shipp TD, Lyons JG, Bromley B. Width of the normal uterine cavity in premenopausal women and effect of parity. Obstet Gynecol 2010;116:305-10.

22. Puente JM, Fabris A, Patel J, Patel A, Cerrillo M, Requena A, Garcia-Velasco JA. Adenomyosis in infertile women: prevalence and the role of $3 \mathrm{D}$ ultrasound as a marker of severity of the disease. Reprod Biol Endocrinol 2016;14:60.

23. Canteiro R, Bahamondes MV, dos Santos Fernandes A, Espejo-Arce X, Marchi NM, Bahamondes L. Length of the endometrial cavity as measured by uterine sounding and ultrasonography in women of different parities. Contraception 2010;81:515-9.

24. Verguts J, Ameye L, Bourne T, Timmerman D. Normative data for uterine size according to age and gravidity and possible role of the classical golden ratio. Ultrasound Obstet Gynecol 2013;42:713-7.

25. Paule SG, Heng S, Samarajeewa N, Li Y, Mansilla M, Webb AI, Nebl T, Young SL, Lessey BA, Hull ML, Scelwyn M, Lim R, Vollenhoven B, Rombauts LJ, Nie $\mathrm{G}$. Podocalyxin is a key negative regulator of human endometrial epithelial receptivity for embryo implantation. Hum Reprod 2021;36:1353-66.

26. Gurung S, Greening DW, Catt S, Salamonsen L, Evans J. Exosomes and soluble secretome from hormone-treated endometrial epithelial cells direct embryo implantation. Mol Hum Reprod 2020;26:510-20.

27. Gilligan LA, Trout AT, Schuster JG, Schwartz BI, Breech LL, Zhang B, Towbin AJ. Normative values for ultrasound measurements of the female pelvic organs throughout childhood and adolescence. Pediatr Radiol 
2019;49:1042-50

28. Gao H, Liu DE, Li Y, Tang J, Hu S, Wu X, Tian Z, Tan H. Uterine size and volume are associated with a higher clinical pregnancy rate in patients undergoing

Cite this article as: Feng Y, Zhang S, Zhou Y, He G, Hong L, Shi L, Wang J, Zhang P, Zhai L. Three-dimensional measurement and analysis of morphological parameters of the uterus in infertile women. Quant Imaging Med Surg 2022;12(4):2224-2237. doi: 10.21037/qims-21-812 assisted reproduction technology: A longitudinal study (A STROBE-compliant article). Medicine (Baltimore) 2019;98:e14366. 